\title{
Photo-activated disinfection in endodontics
}

\author{
Microbiological evaluation of photo-activated disinfection in endodontics (An in vivo study)
}

S. J. Bonsor, R. Nichol, T. M. S. Reid and G. J. Pearson Br Dent J 2006; 200: 337-341

\section{Objective}

To determine the microbiological effect of photoactivated disinfection as an adjunct to normal root canal disinfection in vivo.

Design

A randomised trial carried out in general dental practice.

Subjects and methods

Patients presenting with symptoms of irreversible pulpitis or

periradicular periodontitis requiring endodontic therapy were selected at random. A microbiological sample of the canal was taken on accessing the canal, after conventional endodontic therapy, and finally after the PAD process (photosensitiser and light) had been carried out on the prepared canal. All three samples from each canal were plated within 30 minutes of sampling and cultured anaerobically for five days. Growth of viable bacteria was recorded for each sample to determine bacterial load. Results

Thirty of the 32 canals were included in the results. Cultures from the remaining two did not reach the laboratory within the target time during which viability was sustained. Of the remaining 30, 10 canals were negative to culture. These were either one of the canals in multi rooted teeth where the others were infected or where a pre-treatment with a poly-antibiotic paste had been applied to hyperaemic vital tissue. Sixteen of the remainder were negative to culture after conventional endodontic therapy. Three of the four which had remained infected cultured negative after the PAD process. In the one canal where culturable bacteria were still present, a review of the light delivery system showed a fracture in the fibre reducing the effective light output by $90 \%$. Conclusions

The PAD system offers a means of destroying bacteria remaining after using conventional irrigants in endodontic therapy.

\section{IN BRIEF}

- Bacteria are responsible for the most commonly encountered dental diseases including pulpal pathology.

- The success rate of endodontics relies on the root canal system being rendered bacteria free.

- Conventional chemo-mechanical canal preparation techniques are unable to disinfect the canals predictably and consistently.

- PAD offers potential to eliminate bacteria from the root canals especially where conventional techniques have failed to do so.

\section{COMMENT}

It is generally accepted that the goal of endodontic treatment is to eliminate bacterial pathogens and their by-products from the root canal system and the procedures involved with cleaning and shaping are predominantly associated with addressing this aim.

However, the complexities of the morphology of the root canal system mean that pathogens may reside in locations which are inaccessible to mechanical debridement procedures and that chemical measures, such as irrigation with sodium hypochlorite may not necessarily be $100 \%$ effective. This is an important consideration as studies show that the lower the bacterial contamination in a prepared root canal, the higher the success rate.

The authors of this paper have addressed the above by considering the adjunctive use of PAD to endodontic cleaning and shaping procedures. The principle is that photosensitive molecules attach to the membrane of bacteria. Irradiation with light at a specific wavelength leads to the production of singlet oxygen which causes the bacterial cell wall to rupture. This principle has been applied to endodontic use whereby a small diode laser is attached to a delivery fibre, hand-piece and emitter and used in conjunction with a photosensitiser, tolonium chloride. As well as being shown to be effective in killing bacteria associated with endodontic infections, the PAD system will also kill enterococcus faecaelis, a known contaminant of teeth requiring re-treatment.

From the practical perspective, the canal is cleaned and shaped according to accepted principles. After completion of preparation, it is flooded with the photosensitiser and then irradiated. Filling is then carried out as normal. This paper set out to evaluate the clinical handling of the device and to determine the bacterial load within the canal after initial access, following conventional canal preparation and then following treatment of the canal using the PAD system.

This was a limited study of 32 canals in 14 patients and took place in practice. The method of culture was chosen to assess the bacterial load of common facultative anaerobes and aerobes associated with endodontic infections. It was found that 20 canals were initially infected; after irrigation, four remained infected and following use of the PAD system, only one canal was found to contain cultivable flora.

One of the observed problems associated with the use of the system lay in certain practicalities. The rationale of using this method is that the solution and energy is applied to the bacteria. This involved injection of the PAD solution, placement of an emitter fibre to within $4 \mathrm{~mm}$ of the tooth apex and then moving it up and down by $3 \mathrm{~mm}$ in a vertical direction and at 20 second intervals. This motion was considered to have been responsible for fracture of the fibre tip and has now been discontinued.

This paper addresses a fundamental issue in endodontic procedures, ie the elimination of endodontic pathogens and their by-products, and although this is a limited study, it is significant in that it was carried out in general practice. The findings should be regarded with some caution, in view of the small number of canals treated, but this should not detract from the fact that no measures will remove the need for meticulous cleaning and shaping procedures to ensure the best possible chance of success. Perhaps as significant is the quality of the coronal seal, without which any meticulously prepared canal cannot be expected to remain free from contamination. 